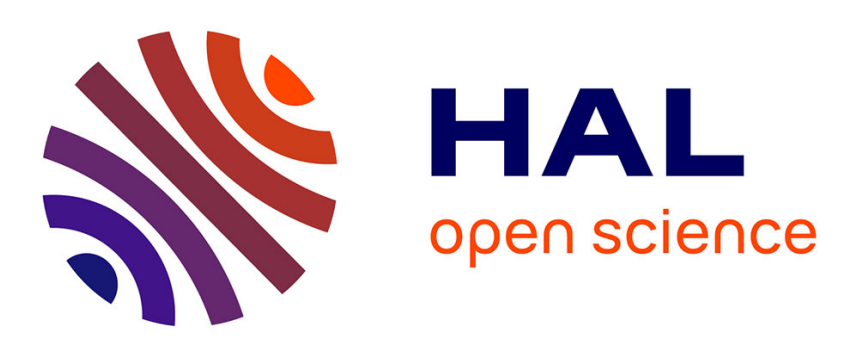

\title{
Half integer quantum Hall effect in high mobility single layer epitaxial graphene
}

Xiaosong Wu, Yike Hu, Ming Ruan, Nerasoa K. Madiomanana, John H. Hankinson, Mike Sprinkle, Claire Berger, Walt. A. de Heer

\section{To cite this version:}

Xiaosong Wu, Yike Hu, Ming Ruan, Nerasoa K. Madiomanana, John H. Hankinson, et al.. Half integer quantum Hall effect in high mobility single layer epitaxial graphene. Applied Physics Letters, 2009, 95 (22), pp.223108. 10.1063/1.3266524 . hal-01002933

\section{HAL Id: hal-01002933 https://hal.science/hal-01002933}

Submitted on 7 Jun 2014

HAL is a multi-disciplinary open access archive for the deposit and dissemination of scientific research documents, whether they are published or not. The documents may come from teaching and research institutions in France or abroad, or from public or private research centers.
L'archive ouverte pluridisciplinaire HAL, est destinée au dépôt et à la diffusion de documents scientifiques de niveau recherche, publiés ou non, émanant des établissements d'enseignement et de recherche français ou étrangers, des laboratoires publics ou privés. 


\title{
Half integer quantum Hall effect in high mobility single layer epitaxial graphene
}

\author{
Xiaosong Wu, ${ }_{1}^{1}$ Yike Hu, ${ }^{1}$ Ming Ruan, ${ }^{1}$ Nerasoa K Madiomanana, ${ }^{1}$ John Hankinson, ${ }^{1}$ \\ Mike Sprinkle, ${ }^{1}$ Claire Berger, ${ }^{1,2}$ and Walt A. de Heer ${ }^{1, a)}$ \\ ${ }^{1}$ School of Physics, Georgia Institute of Technology, Atlanta, Georgia 30332, USA \\ ${ }^{2}$ CNRS-Institut Néel, BP 166, 38042 Grenoble, Cedex 9, France
}

(Received 15 September 2009; accepted 26 October 2009; published online 4 December 2009)

\begin{abstract}
The quantum Hall effect, with a Berry's phase of $\pi$ is demonstrated here on a single graphene layer grown on the $\mathrm{C}$-face of $4 \mathrm{H}$ silicon carbide. The mobility is $\sim 20000 \mathrm{~cm}^{2} / \mathrm{V} \cdot \mathrm{s}$ at $4 \mathrm{~K}$ and $15000 \mathrm{~cm}^{2} / \mathrm{V} \cdot \mathrm{s}$ at $300 \mathrm{~K}$ despite contamination and substrate steps. This is comparable to the best exfoliated graphene flakes on $\mathrm{SiO}_{2}$ and an order of magnitude larger than Si-face epitaxial graphene monolayers. These and other properties indicate that $\mathrm{C}$-face epitaxial graphene is a viable platform for graphene-based electronics. (C) 2009 American Institute of Physics. [doi:10.1063/1.3266524]
\end{abstract}

In 2004 Berger et al. ${ }^{1}$ introduced the concept of graphene-based electronics and a route to realize it. Results in that paper on patterned epitaxial graphene grown on the $\mathrm{Si}$-face of $4 \mathrm{H}$ silicon carbide crystals showed that the material could be top-gated and it also showed magnetoresistance measurements with characteristic Shubnikov de Haas oscillations (SdHOs). In one sample (sample A of Ref. 1), the SdHOs corresponding to the $n=2$ and $n=3$ Landau levels were observed as well as a weak modulation in the Hall effect. The quantum Hall effect (QHE) was not observed, at least not for these relatively large index Landau levels. The mobility was also relatively low $\left(\mu=1100 \mathrm{~cm}^{2} / \mathrm{V} \cdot \mathrm{s}\right)$. From low-energy electron diffraction and Auger electron spectroscopy we estimated that the material was three monolayers, but we now know, from better understanding of the structure $^{2}$ and the low field linearity of the Hall effect, that it was in fact a single layer. We also have found that relatively low mobilities are characteristic for Si-face epitaxial graphene compared with the $\mathrm{C}$-face.

Subsequent work focused on multilayered epitaxial graphene (MEG) grown on the carbon face of silicon carbide which demonstrated high mobilities. To compare with previous work, a summary of the properties of MEG is provided, although the experiments were performed on single layer epitaxial graphene (SEG).

In epitaxial graphene, the graphene layer at the interface with the substrate is n-doped due to the Schottky barrier at the interface, while the other layers are essentially undoped. The individual layers of MEG are electronically decoupled due to an unusual rotational order ${ }^{2}$ and consequently the layers exhibit graphene properties rather than graphitic properties as might be expected. ${ }^{3-10}$ Specifically, the interface layer, which carries most of the current, shows the nontrivial Berry's phase and has a high mobility $\left(>30000 \mathrm{~cm}^{2} / \mathrm{V} \cdot \mathrm{s}\right)$ while the other, undoped layers have even much higher mobilities $\left(>250000 \mathrm{~cm}^{2} / \mathrm{V} \cdot \mathrm{s}\right)$. Moreover, at least the top-most layer has been shown to be continuous over the entire surface, without breaks or crystallographic domain boundaries. ${ }^{9}$ Nevertheless, the QHE is not seen. ${ }^{11,13}$

Many applications, including terahertz electronics, ${ }^{12}$ require room temperature mobilities of the order of

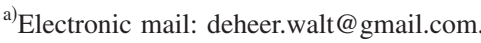

$10000 \mathrm{~cm}^{2} / \mathrm{V} \cdot \mathrm{s}$. We have demonstrated patterned samples with $\mu \sim 30000 \mathrm{~cm}^{2} / \mathrm{V} \cdot \mathrm{s}$ in multilayer epitaxial graphene grown on the $\mathrm{C}$-face of hexagonal $\mathrm{SiC}$ (MEG) using the so-called rf furnace method, ${ }^{3,13,14}$ in which the substrate is enclosed in a graphitic chamber with or without inert gas to produce high quality graphene of arbitrary thickness. Although challenging we succeeded in producing SEG on the $\mathrm{C}$-face of $\mathrm{SiC}$ (it is easier to produce SEG on the Si-face $\left.{ }^{15,16}\right)$.

Here we show the QHE in SEG in two samples (sample A and sample B). The inset of Fig. 1(a) shows an atomic force microscopy image (AFM) of sample A, a Hall bar that was deliberately patterned ${ }^{3}$ over steps on the substrate surface in order to evaluate the effects of steps on the transport properties. The AFM image shows e-beam resist residue particles from the processing and characteristic pleats (white lines) on the graphene surface. ${ }^{2}$

The mobility of the sample is $20000 \mathrm{~cm}^{2} / \mathrm{V} \cdot \mathrm{s}$. The QHE is well resolved in Fig. 1(a), which shows quantum Hall plateaus in the magnetic field dependence of the Hall resistance, as first observed in exfoliated graphene flakes on $\mathrm{SiO}_{2}{ }^{17,18}$ The Hall plateaus correspond to transverse resistances $\rho_{x y}=\left(h / 4 e^{2}\right) /(n+1 / 2)$ for $n=0$ to 3, where $n$ is the Landau level index, which establishes the nontrivial Berry's phase of $\pi .{ }^{19}$ The longitudinal resistivity $\rho_{x x}$ [Fig. 1(a)] shows the characteristic SdHOs, in which Landau levels from $n=0$ upto $n=8$ are easily recognized. The SdHOs develop into the QHE in high fields, manifested by characteristic zero resistance minima and Hall plateaus. The graphene charge density obtained from the Hall coefficient (temperature independent) is found to be $n_{s}=1.27 \times 10^{12} / \mathrm{cm}^{2}$ (hole doped) and temperature independent. The graphene layer is negatively doped due to the work function difference at the $\mathrm{SiC}$ graphene interface. ${ }^{3}$

The graphene surface has accumulated its positive charge from environmental humidity. The charge density can be controlled by adjusting the exposure to humidity as well as by exposure to ambient light. Note that epitaxial graphene surfaces can be immaculately cleaned by heating in vacuum to $1000{ }^{\circ} \mathrm{C} .{ }^{9}$ Also the conventional (local) top gating methods used for applications ${ }^{20}$ cannot be used to demonstrate the QHE. 


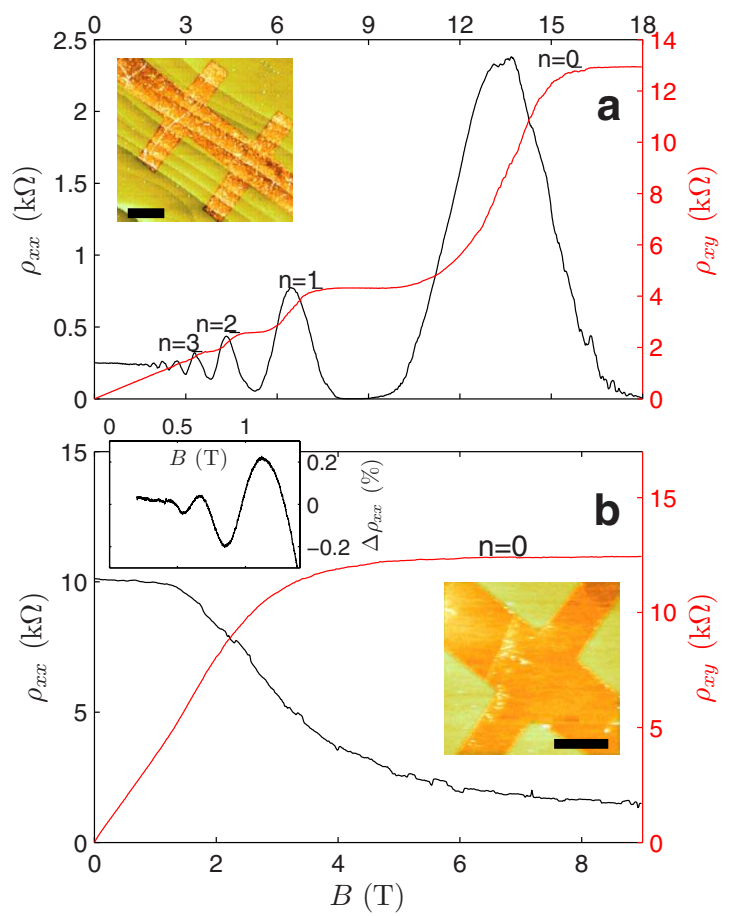

FIG. 1. (Color online) Quantum Hall effect in two monolayer epitaxial graphene samples measured at $1.4 \mathrm{~K}$. Panel a, Sample A. Hall resistance (red) as a function of magnetic field showing characteristic Hall plateaus at $\rho_{x y}=\left(h / 4 e^{2}\right) /(n+1 / 2)$, where $n$ is the Landau level index, magnetoresistivity $\rho_{x x}$ (black) showing characteristic oscillations and dropping to zero for low Landau indexes. Intriguing reproducible fine structure features are observed in both $\rho_{x x}$ and $\rho_{x y}$ in high fields. Inset: AFM image of the Hall bar $(1.8 \mu \mathrm{m} \times 4.6 \mu \mathrm{m})$ patterned over several $\mathrm{SiC}$ steps, showing e-beam resist residue particles (white spots, covering about $17 \%$ of the surface) and pleats in the graphene (white lines). Panel b, Sample B. Red: Hall resistance showing a Hall plateau for $n=0$. Black: magnetoresistance $\rho_{x x}$. Very weak oscillations can be discerned at $n=1,2,3$ (upper inset). Lower Inset: AFM image of the Hall cross $(1.5 \mu \mathrm{m} \times 2.5 \mu \mathrm{m})$. The substrate is step-free and the surface is clean. The scale bars represent $2 \mu \mathrm{m}$.

The second Hall bar (sample B) was deliberately patterned on a step-free terrace, Fig. 1(b); its mobility is $4000 \mathrm{~cm}^{2} / \mathrm{V} \cdot \mathrm{s}$. It also exhibits the QHE, however only a single quantum Hall plateau corresponding to the $n=0$ Landau level is observed. The SdHOs corresponding to the other Landau levels are very small and reminiscent of those seen in MEG. ${ }^{5,13}$

Sample A was measured three times after re-exposing it to conditions with different humidity. The charge densities $n_{s}$ are $0.9,1.28$, and $1.27 / \mathrm{cm}^{2}$, while the mobility varies slightly $(\sim 5 \%$ at $4.2 \mathrm{~K}$.). The QHE is observed for all three experimental runs.

Despite the fact that the graphene in sample A is draped over several steps, is heavily contaminated (cf. the hole doping and the particles) and has pleats, the mobility is as high as $20000 \mathrm{~cm}^{2} / \mathrm{V} \mathrm{s}$ at $4.2 \mathrm{~K}$ and $15000 \mathrm{~cm}^{2} / \mathrm{V} \cdot \mathrm{s}$ at $300 \mathrm{~K}$ and shows only a mild temperature dependence (similar to MEG samples ${ }^{3}$ ). These observations show that (1) scattering from impurities is weak, (2) electron-phonon scattering is suppressed, ${ }^{6}$ and (3) the graphene is continuous over steps in the $\mathrm{SiC}$ substrate.

For the case where the charge density $n_{s}$ is 1.28 $\times 10^{12} / \mathrm{cm}^{2}$, the experiment has been carried out in magnetic fields up to $9 \mathrm{~T}$ and at temperatures up to $150 \mathrm{~K}$, where SdHOs for the $n=1$ Landau level can still be seen. The temperature dependence of SdHOs is plotted in Fig. 2. The

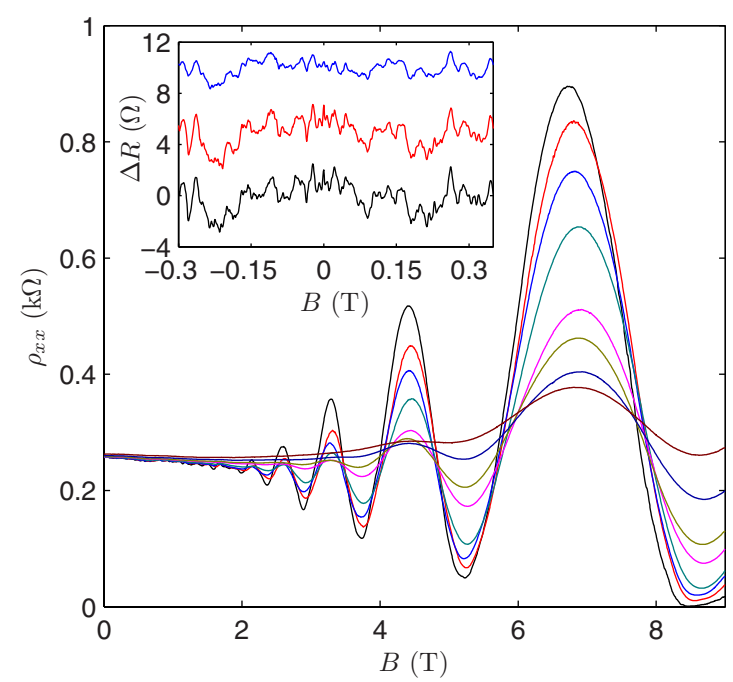

FIG. 2. (Color online) Temperature dependence of the magnetoresistance. Main panel, SdHOs at different temperatures, 1.4, 10, 20, 30, 50, 60, 80, and $100 \mathrm{~K}$. Inset: low field magnetoresistance at $4.2,10$, and $30 \mathrm{~K}$ exhibits universal conductance fluctuations, indicating that the phase coherence length $l_{\phi}$ is comparable to the size of the sample. Data are shifted in $y$-axis for clarity.

damping of the oscillations with temperature is caused by thermal broadening of Landau levels. In graphene, the temperature dependence of the amplitude is described by $t_{k} / \sinh t_{k}$, where $t_{k}=2 \pi^{2} k_{B} T \mu / \hbar v_{0}^{2} e B .^{21}$ Here, $\mu, T, B$, and $v_{0}$ are chemical potential, temperature, magnetic field, and the band velocity, respectively. Using this formula, we find the velocity $v_{0}=1.14 \times 10^{6} \mathrm{~m} / \mathrm{s}$, which agrees with the $v_{0}$ of graphene flakes on $\mathrm{SiO}_{2},{ }^{17,18}$ which, combined with the graphene Berry's phase establishes that the $\mathrm{SiC}$ substrate does not affect the properties of EG any more than the $\mathrm{SiO}_{2}$ substrate affects exfoliated graphene.

We have also investigated the low field magnetoresistance. As shown in the inset of Fig. 2, the sample displays aperiodic and reproducible universal conductance fluctuations, that diminish with increasing temperatures. The phase coherence length is estimated from the magnetoconductance correlation function; $F(\Delta B)=\langle[G(B)-\langle G(B)\rangle][G(B+\Delta B)$ $-\langle G(B+\Delta B)\rangle]\rangle .^{22}$ A correlation field $\Delta B_{c}$ is defined as the half-width at half-height $F\left(\Delta B_{c}\right)=F(0) / 2$. The phase coherence length $l_{\phi}$ is related to $\Delta B_{c}$ by $\Delta B_{c} \approx e / h l_{\phi}^{2}$. We find $l_{\phi}$ $\approx 0.6 \mu \mathrm{m}$ at $4.2 \mathrm{~K}$, similar to values previously found in MEG ribbons. ${ }^{3,5}$

Besides the QHE, we have shown that (1) SEG can be grown on the $\mathrm{C}$-face of hexagonal silicon carbide wafers; (2) the graphene sheet is continuous over substrate steps; (3) its mobility rivals that of the best exfoliated graphene on $\mathrm{SiO}_{2}$, despite significant contamination, substrate steps, and harsh processing procedures; (4). the QHE in C-face epitaxial graphene demonstrates that the substrate is at least as unimportant here as it is for exfoliated graphene on $\mathrm{SiO}_{2}$.

Concluding, the robustness and large scale patterning that is possible with epitaxial graphene opens new avenues for graphene physics. This important development brings epitaxial graphene yet a step closer to becoming a scalable platform for graphene-based electronics as anticipated. ${ }^{1,23}$

This work was supported by NSF under Grant No. DMR-0820382 and the W. M. Keck Foundation. A portion of this work was performed at the National High Magnetic 
Field Laboratory, which is supported by NSF Cooperative Agreement under Grant No. DMR-0654118, by the State of Florida, and by the DOE. We would like to thank Z. G. Jiang for insightful discussions. We also acknowledge E. C. Palm, T. P. Murphy, J.-H. Park, G. E. Jones, and Z. L. Guo for experimental assistance.

${ }^{1}$ C. Berger, Z. M. Song, T. B. Li, X. Li, A. Y. Ogbazghi, R. Feng, Z. Dai, A. N. Marchenkov, E. H. Conrad, P. N. First, and W. A. de Heer, J. Phys. Chem. B 108, 19912 (2004)

${ }^{2}$ J. Hass, F. Varchon, J. E. Millán-Otoya, M. Sprinkle, N. Sharma, W. A. de Heer, C. Berger, P. N. First, L. Magaud, and E. H. Conrad, Phys. Rev. Lett. 100, 125504 (2008).

${ }^{3}$ C. Berger, Z. M. Song, X. B. Li, X. Wu, N. Brown, C. Naud, D. Mayou, T. Li, J. Hass, A. N. Marchenkov, E. H. Conrad, P. N. First, and W. A. de Heer, Science 312, 1191 (2006)

${ }^{4}$ M. L. Sadowski, G. Martinez, M. Potemski, C. Berger, and W. A. de Heer, Phys. Rev. Lett. 97, 266405 (2006).

${ }^{5}$ X. S. Wu, X. B. Li, Z. M. Song, C. Berger, and W. A. de Heer, Phys. Rev. Lett. 98, 136801 (2007).

${ }^{6}$ M. Orlita, C. Faugeras, P. Plochocka, P. Neugebauer, G. Martinez, D. K. Maude, A.-L. Barra, M. Sprinkle, C. Berger, W. A. de Heer, and M. Potemski, Phys. Rev. Lett. 101, 267601 (2008).

${ }^{7}$ P. Plochocka, C. Faugeras, M. Orlita, M. L. Sadowski, G. Martinez, M. Potemski, M. O. Goerbig, J.-N. Fuchs, C. Berger, and W. A. de Heer, Phys. Rev. Lett. 100, 087401 (2008).

${ }^{8}$ D. Sun, Z. K. Wu, C. Divin, X. Li, C. Berger, W. A. de Heer, P. N. First, and T. B. Norris, Phys. Rev. Lett. 101, 157402 (2008).

${ }^{9}$ D. L. Miller, K. D. Kubista, G. M. Rutter, M. Ruan, W. A. de Heer, P. N. First, and J. A. Stroscio, Science 324, 924 (2009).

${ }^{10}$ M. Sprinkle, D. Siegel, Y. Hu, J. Hicks, P. Soukiassian, A. Tejeda, A.
Taleb-Ibrahimi, P. Le Fèvre, F. Bertran, C. Berger, W. A. de Heer, A. Lanzara, and E. H. Conrad, arXiv:0907.5222v1 (unpublished).

${ }^{11}$ P. Darancet, N. Wipf, C. Berger, W. A. de Heer, and D. Mayou, Phys. Rev. Lett. 101, 116806 (2008)

${ }^{12}$ J. Moon, D. Curtis, M. Hu, D. Wong, C. McGuire, P. M. Campbell, G. Jernigan, J. L. Tedesco, B. VanMil, R. Myers-Ward, C. Eddy, and D. K. Gaskill, IEEE Electron Device Lett. 30, 650 (2009).

${ }^{13}$ W. A. de Heer, C. Berger, X. S. Wu, P. N. First, E. H. Conrad, X. Li, T. Li, M. Sprinkle, J. Hass, M. L. Sadowski, M. Potemski, and G. Martinez, Solid State Commun. 143, 92 (2007).

${ }^{14}$ J. Hass, W. A. de Heer, and E. H. Conrad, J. Phys.: Condens. Matter 20, 323202 (2008).

${ }^{15}$ K. V. Emtsev, A. Bostwick, K. Horn, J. Jobst, G. L. Kellogg, L. Ley, J. L. McChesney, T. Ohta, S. A. Reshanov, J. Röhrl, E. Rotenberg, A. K. Schmid, D. Waldmann, H. B. Weber, and T. Seyller, Nature Mater. 8, 203 (2009).

${ }^{16}$ C. Virojanadara, M. Syväjarvi, R. Yakimova, L. I. Johansson, A. A. Zakharov, and T. Balasubramanian, Phys. Rev. B 78, 245403 (2008).

${ }^{17}$ Y. B. Zhang, Y. W. Tan, H. L. Stormer, and P. Kim, Nature (London) 438 , 201 (2005).

${ }^{18}$ K. S. Novoselov, A. K. Geim, S. V. Morozov, D. Jiang, M. I. Katsnelson, I. V. Grigorieva, S. V. Dubonos, and A. A. Firsov, Nature (London) 438, 197 (2005).

${ }^{19}$ T. Ando, T. Nakanishi, and R. Saito, J. Phys. Soc. Jpn. 67, 2857 (1998).

${ }^{20}$ J. Kedzierski, P. L. Hsu, P. Healey, P. W. Wyatt, C. L. Keast, M. Sprinkle, C. Berger, and W.A. de Heer, IEEE Trans. Electron Devices 55, 2078 (2008).

${ }^{21}$ V. P. Gusynin and S. G. Sharapov, Phys. Rev. B 71, 125124 (2005).

${ }^{22}$ C. W. J. Beenakker and H. van Houten, Solid State Phys. 44, 1 (1991).

${ }^{23}$ W. A. de Heer, C. Berger, and P. N. First, U.S. Patent No. $7015142(21$ March 2006). 